\title{
Condiciones socioeconómicas e higiénico-sanitarias como dimensiones de la seguridad alimentaria y nutricional
}

\author{
Socioeconomic and sanitary \\ conditions as dimensions of food \\ and nutrition security
}

\begin{abstract}
The objective of this work was to know the socioeconomic and sanitary conditions of households in Chapecó (SC) as dimensions of Food and Nutrition Security. We surveyed 774 households from urban and rural areas. The majority of households heads are women $(68.8 \%) ; 8.4 \%$ of the households heads are illiterate, $62.4 \%$ have primary education, $20.93 \%$ high school and $7.8 \%$ have higher education (incomplete/full, respectively); The average family monthly income was 1.803,8 dollars. The water supply in urban area is done by the public system (84\%) and in rural area comes from wells/sources (50.2\%). The public sewer system exists only in urban areas (23.4\%); $75.4 \%$ of the households conduct sewage for tanks in urban area and $99 \%$ in rural area. It is considered the household incomes observed facilitate the access to food; however the level of education of more than $70 \%$ of household heads is equal to or less than primary education. This may imply the lack of knowledge about hygiene care and food handling. The large number of families who conduct sewage for tanks and that use water from sources/wells for drinking water may prejudice their Food and Nutrition Security condition. Key words: Socioeconomic indicators, food and nutrition security, urban, rural.
\end{abstract}

\section{Maria Assunta B. (1) \\ Luciara Souza G.(2) \\ Carla Rosane Paz Arruda Teo (1) \\ Marcia Pozzagnol (1)}

(1) Programa de Pós-graduación stricto sensu en Ciencias de la Salud. Universidade Comunitária da Região de Chapecó (UNOCHAPECÓ), Chapecó-SC, Brasil.

(2) Carrera de Nutrición. Universidade Comunitária da Região de Chapecó (UNOCHAPECÓ) Chapecó-SC, Brasil.

Dirigir la correspondência a: Profesora

María Assunta Busato Av. Attílio Fontana 591E, EFAPI, 89809000 Chapecó-SC, Brasil Fone (55) 4933218215 FAX (55) 4933218215 e.mail: assunta@unochapeco.edu.br

Este trabajo fue recibido el 18 de Noviembre de 2015 y aceptado para ser publicado el 14 de Enero de 2016.

\section{INTRODUCCIÓN}

La Seguridad Alimentaria y Nutricional (SAN) es uno de los derechos humanos básicos e incluye asegurar el acceso a alimentos seguros y de calidad, en cantidad suficiente y de forma permanente, sin comprometer el acceso a otras necesidades esenciales y con base en prácticas de alimentación saludables. En este sentido, la SAN intrafamiliar se logra cuando todos sus miembros tienen este derecho (1). Sin embargo, la SAN y (in) SAN son conceptos complejos para ser evaluados y aceptados como variables latentes (2).

Teniendo en cuenta la alimentación como un derecho, al que se debe garantizar el acceso, tanto desde el punto de vista de la seguridad como la cantidad y la calidad, garantizar una alimentación adecuada para todos los ciudadanos es un gran desafío. Mientras la promoción y la garantía de la SAN están siendo incorporadas a los planes estratégicos de los gobiernos, los estudios sobre las condiciones higiénicas y sobre las prácticas de manipulaciones y preparación de alimentos se llevan a cabo en todo el mundo. Entre ellos, destacamos la preocupación por la calidad sanitaria de los alimentos comercializados (3). Sin embargo, las condiciones higiénico-sanitarias que implican la manipulación de los alimentos durante la producción, almacenamiento, procesamiento, distribución o manipulación en los hogares también pueden suponer un riesgo para la salud humana.

La calidad higiénico-sanitaria como factor de la SAN ha sido ampliamente estudiada y discutida, puesto que las enfermedades transmitidas por los alimentos uno de los principales factores que contribuyen a los índices de morbilidad en los países de la América Latina y del Caribe (4). Para el Comité de Agricultura y Alimentación de la Organización de las Naciones Unidas (FAO), las enfermedades que se derivan de alimentos contaminados pueden ser el mayor problema de salud en el mundo contemporáneo (4). Las enfermedades transmitidas por los alimentos son un importante problema de salud pública (5).

Este estudio tuvo como objetivo comprender las condiciones socioeconómicas, higiénico-sanitarias y el saneamiento 
básico del ambiente de los hogares de la población en las zonas urbana y rural del municipio de Chapecó (SC), suponiendo que estas variables constituyen las dimensiones de la SAN, como explicó el concepto de este fenómeno.

\section{SUJETOS Y MÉTODOS}

Este estudio se caracterizó por un enfoque cuantitativo, de carácter exploratorio descriptivo, con delineamiento transversal de base poblacional. Para el planeamiento de la muestra, se utilizaron datos del Departamento Municipal de Salud (DMS), cuya población es de 200 mil habitantes, estando 50.286 familias/hogares en la zona urbana y 2.977 familias/hogares en la zona rural de Chapecó (SC), distribuidos en cuatro áreas que constituyen los territorios de los Núcleos de Apoyo para la Salud de la Familia (NASF), implantados en el municipio.

El diseño de la muestra comportó de dos fases, realizándose un proceso de muestreo estratificado proporcional en la que los estratos fueron los territorios de los cuatro NASF, seguido de un proceso de muestreo aleatorio sistemático de hogares.

Se optó por el hogar como unidad de análisis de interés por el hecho de que la SAN es medida, tradicionalmente, como una condición intrafamiliar (6). Para una prevalencia conocida de la inseguridad alimentaria de $16,8 \%$ en el estado de Santa Catarina (7), un nivel de significación del 5,0\% y un error aceptable del $5,0 \%$, con aumento del $10,0 \%$ por las pérdidas y rechazos, y aplicación del factor 2,0 para compensación del efecto de diseño de la muestra, el tamaño de la muestra se fijó en 559 hogares de la zona urbana y 215 en la rural, proporcionalmente distribuidos también entre los territorios de los NASF. Las entrevistas fueron realizadas con los jefes de familia. Se consideró como jefe de familia aquella persona que fue designada por el grupo familiar.

La recolección de los datos se realizó en el periodo entre septiembre de 2011 y agosto de 2012. La entrevista fue realizada durante una visita domiciliar, en un sólo encuentro, tanto en la zona urbana como en la rural. Para conocer las condiciones higiénico-sanitarias de manipulación de alimentos en los hogares, el instrumento utilizado fue adaptado de Piragine (8) (2005) que fue previamente validada. Para contemplar variables socioeconómicas, demográficas y de saneamiento fue utilizado cuestionario construido por los investigadores. El análisis de los datos fue realizado mediante estadística descriptiva, siendo los resultados presentados como frecuencias (absoluta y relativa). Los encuestados fueron informados sobre los objetivos y procedimientos de la investigación y se les pidió su aceptación para participar en el estudio mediante la firma del Formulario de Consentimiento Libre y Aclarado. El proyecto que dio origen a este trabajo fue sometido a la apreciación del Comité de Ética de Investigación de la Universidad Comunitária de la Región de Chapecó (UNOCHAPECÓ) y recibió el parecer de aprobación nº 080/2010.

\section{RESULTADOS Y DISCUSIÓN}

A pesar de la influencia de las condiciones económicas en la polarización del perfil social de la población (9), otras condiciones pueden ser consideradas como indicadores de la

\section{TABLA 1}

Características socioeconómicas de los jefes de familia de la población urbana y rural de Chapecó (SC).

\begin{tabular}{|c|c|c|c|c|c|c|}
\hline \multirow[t]{2}{*}{ Variables } & \multicolumn{2}{|c|}{ Población urbana } & \multicolumn{2}{|c|}{ Población rural } & \multicolumn{2}{|c|}{ Total } \\
\hline & $n$ & $\%$ & $n$ & $\%$ & $\mathrm{n}$ & $\%$ \\
\hline \multicolumn{7}{|l|}{ Jefe de la familia } \\
\hline Hombre & 200 & 37,96 & 41 & 19 & 241 & 31,14 \\
\hline Mujer & 359 & 62,09 & 174 & 81 & 533 & 68,86 \\
\hline \multicolumn{7}{|l|}{ Color auto declarada } \\
\hline Blanca & 415 & 74,24 & 141 & 65,6 & 556 & 71,83 \\
\hline Mestiza & 136 & 24,33 & 68 & 31,6 & 204 & 26,35 \\
\hline Negra & 8 & 1,43 & 6 & 2,8 & 14 & 1,8 \\
\hline \multicolumn{7}{|l|}{ Escolaridad } \\
\hline Sin escolaridad & 45 & 8,05 & 20 & 9,3 & 65 & 8,4 \\
\hline \multicolumn{7}{|l|}{ Primaria } \\
\hline Incompleto/Completo & 332 & 59,39 & 151 & 70,24 & 483 & 62,4 \\
\hline Secundaria Incompleto/Completo & 126 & 22,54 & 36 & 16,75 & 162 & 20,93 \\
\hline Superior Incompleto/Completo & 53 & 9,48 & 8 & 3,7 & 61 & 7,88 \\
\hline No sabe & 3 & 0,53 & - & - & 3 & 0,39 \\
\hline \multicolumn{7}{|l|}{ Ocupación } \\
\hline Tiene empleo & 241 & 43,11 & 88 & 40,93 & 329 & 42,5 \\
\hline Desempleado & 43 & 7,69 & 4 & 1,87 & 47 & 6,07 \\
\hline Dueña de casa & 101 & 18,07 & 51 & 23,7 & 152 & 19,64 \\
\hline Jubilado/Pensionista & 174 & 31,13 & 67 & 31,17 & 241 & 31,14 \\
\hline \multirow[t]{2}{*}{ Tiene empleo/Jubilado } & - & - & 5 & 2,3 & 5 & 0,65 \\
\hline & \multicolumn{2}{|c|}{ Población urbana } & \multicolumn{2}{|c|}{ Población rural } & \multicolumn{2}{|c|}{$\begin{array}{c}\text { Media } \\
\text { (urbana y rural) }\end{array}$} \\
\hline Renta media del jefe de la familia (dólares) & \multicolumn{2}{|c|}{889,4} & \multicolumn{2}{|c|}{702,8} & \multicolumn{2}{|c|}{796,1} \\
\hline Renta media familiar (dólares) & \multicolumn{2}{|c|}{$2.002,8$} & \multicolumn{2}{|c|}{$1.598,5$} & \multicolumn{2}{|c|}{$1.803,8$} \\
\hline
\end{tabular}


SAN, como los hábitos de higiene en la manipulación de los alimentos y el saneamiento básico. En esta perspectiva, este estudio investigó a 774 hogares, totalizando 2.453 personas, pertenecientes a las áreas urbana y rural, siendo las entrevistas realizadas, en cada hogar, con el jefe de la familia. Las características socioeconómicas de los jefes de familias que integraran la población del estudio (tabla 1) indicaron que la mayoría de los hogares tienen como responsable la figura de la mujer, en la zona urbana (62\%) y sobre todo en la zona rural (81\%), con edad media de 49,3 años (desviación estándar $15,75)$ y el $71,83 \%$ eran de raza blanca. Según Siliprandi (10), las mujeres son más vulnerables a la Inseguridad Alimentaria y Nutricional (IAN), presentando mayor probabilidad de ser pobres a la medida en que la renta de las mujeres es inferior a la de los hombres. En esta lógica, el creciente número de hogares encabezados por mujeres, con renta menor que los encabezados por hombres, resulta en una mayor vulnerabilidad intrafamiliar a la IAN. En el mismo sentido, el estudio de Marín-León (11), desarrollado a partir de los datos de la Investigación Nacional por Muestra de Domicilios (INAD) de 2004, concluyó que las condiciones socioeconómicas más precarias están asociadas a la IAN, y se agrava en los hogares dirigidos por mujeres negras.

La renta media familiar de la población urbana fue $2.002,8$ y de la población rural de 1.598,5 dólares, lo que caracteriza que hay posibilidad de un acceso regular a alimentos de calidad y en cantidad suficiente. Estas condiciones están de acuerdo a lo que afirma Salles-Costa (12) que, la renta familiar mensual per cápita es el indicador que mejor discrimina a las familias en riesgo de la SAN, teniendo en cuenta que, a medida que la renta familiar aumenta, la proporción de familias en la seguridad alimentaria también aumenta. Se considera que la desigualdad de renta sea el factor más importante para determinar la restricción alimentaria, con contribuciones significativas de factores como el bajo nivel de educación, de color negro y residir en ambiente urbano (11). Se infiere que la población del municipio estudiado presenta relativamente baja vulnerabilidad a la condición de la IAN tanto en el espacio urbano como en el rural, sobre todo cuando se tienen en cuenta las variables renta familiar y la residencia en zona urbana.
Por otro lado, para el factor educación se identificó que $70,8 \%$ de los jefes de familia tenían sólo educación primaria y sin escolaridad, lo que puede ser un indicador importante de la vulnerabilidad para la IAN, además de la renta, teniendo en cuenta que la prevalencia de la seguridad alimentaria crece de acuerdo con el grado de educación del jefe de la familia (12) y es un factor protector para la SAN familiar (13).

Sin embargo, aun que la renta sea reconocida como un determinante fundamental de la inseguridad alimentaria, en la actualidad, este factor, por sí solo es un indicador indirecto y limitado de esta condición. Por lo tanto, es necesario identificar cuáles son los grupos más vulnerables, más allá de la cuestión de la renta, debido a otros problemas de la inseguridad alimentaria (14).

El conocimiento de los principales determinantes de la salud, como las condiciones de saneamiento y de vivienda son de gran importancia en el establecimiento de medidas para promover la calidad de vida de las familias. Así, hay que considerar el entorno en el que las familias viven, reconociendo las variables que directa o indirectamente afectan a su bienestar y desarrollo (15). En la tabla 2 se presentan los datos sobre el saneamiento básico en relación con los hogares rurales y urbanos.

El agua puede ser un vehículo importante de agentes biológicos y químicos potencialmente nocivos para el hombre cuando hay una falta de atención y un tratamiento eficaz, poniendo en peligro la salud y el bienestar de una comunidad (14). Amaral (16) considera que el agua para el consumo humano es uno de los vehículos importantes de enfermedades diarreicas de naturaleza infecciosa, que torna primordial la evaluación primaria de su calidad microbiológica.

Este aspecto es especialmente relevante si se considera que este tipo de delito, que se deriva de la mala calidad del agua de consumo, por ejemplo, interfiriere perjudicialmente en la utilización o aprovechamiento de los nutrientes presentes en los alimentos que se accede, caracterizando condición de IAN en el ámbito de la dimensión de vida y salud.

El cuidado de la calidad del agua es esencial para minimizar la posibilidad de transmisión de agentes patogénicos. Amorim et al. (17) consideran que, a pesar de la gran expansión de la disponibilidad de agua potable y saneamiento bá-

\section{TABLA 2}

Saneamiento básico en los hogares de las familias urbanas y rurales del municipio de Chapecó (SC).

Población urbana (\%)

84,08

13,62

2,33

23,44

75,48

0,45

0,63

96,81

0,49

2,70
Población rural (\%)
45,11

50,23

4,65

99,06

0,94

90,7

9,30

Elaboración de los autores 
sico en las últimas décadas, los servicios presentan una clara relación con la salud. Este crecimiento ha sido insuficiente para satisfacer las crecientes demandas de la población, en función de urbanización. Teniendo en cuenta esto, se puede considerar que la población de este estudio se presenta vulnerable dado que más del $13 \%$ de la población urbana y la mitad de la rural utilizan agua de pozos o fuentes que, posiblemente, no tienen ningún tipo de tratamiento o control de su calidad.

El sistema de destino de las aguas residuales de las residencias es un factor importante en el cuidado del medio ambiente. Cuando se depositan en fosas puede contaminar a la capa freática y pozos de agua subterránea y de abastecimiento de agua. Esta condición puede poner en peligro la salud de la población, teniendo en cuenta el gran número de familias que tienen la red cloacal destinada a fosas $y$, también, aquellos que utilizan agua de fuente o de pozos sin tratamiento (tabla 2). Además de la posibilidad de contaminación del agua, hay la presencia de insectos y animales en los hogares. Aunque el destino de la basura y la cloaca abierta fue observado en pocos hogares, muchas familias relataron la presencia de insectos como cucarachas $(22,25 \%)$, moscas $(36,39 \%)$, hormigas $(25,54 \%)$ y roedores $(4,43 \%)$. A pesar de este escenario, la mayoría de las familias $(51,16 \%)$ no hace el control de plagas en los hogares. Las condiciones higiénico-sanitarias destacan la vulnerabilidad de las familias en términos de la salud y de la SAN, teniendo en cuenta que tanto el agua y los alimentos pueden ser contaminados por las aguas residuales, basura, insectos y animales que vagan por el sistema residencial.

Otro factor determinante de la SAN considerado es la condición de almacenamiento y conservación de los alimentos en los que se deben observar los factores ambientales como la temperatura, la humedad, el tiempo de espera fuera de refrigeración después del cocimiento y antes de su consumo, además de lavado e higienizado de alimentos tales como frutas, verduras y legumbres (18). Para Oliveira (19), los riesgos de contaminación son mayores cuando la preparación de alimentos se hace con mucha antelación, lo que favorece la exposición prolongada. Aún, las condiciones de falta de higiene en la preparación y distribución también contribuyen para esto. Los principales problemas son consecuencias del recalentamiento y refrigeración inadecuados (4).

Este estudio identificó que en la mayoría de los hogares, tanto el almuerzo $(88,98 \%)$ como la cena $(72,09 \%)$ son preparados al momento de la comida. En relación a las sobras, la mayoría de las familias $(52,44 \%)$ guarda en la nevera, pero hay una parte $(48,57 \%)$ que informa nunca sobrar alimento. Se percibió que las familias tenían cuidados con la conservación de los alimentos preparados, lo que evita, en alguna medida, la propagación y proliferación de microorganismos patógenos, minimizando los riesgos de aparición de enfermedades transmitidas por alimentos. El uso de huevos crudos en la preparación de recetas fue relatado por $43,7 \%$ de las familias. Cuando descongelan carnes y productos similares, el proceso se realiza en vasija con agua $(46,9 \%)$, en el mostrador $(22,73 \%)$ o en la nevera $(20,22 \%)$. El uso de huevos crudos y descongelación de los alimentos son las prácticas que merecen atención, pues pueden poner en peligro la salud de los alimentos.

El estudio también muestra que la mayoría de las familias realiza la higiene de las frutas y las verduras antes de comer (tabla 3). El hábito de comer frutas y verduras es benéfico, ya que son ricas en vitaminas y minerales esenciales para la salud. Sin embargo, si no hay un saneamiento adecuado aumenta el riesgo de contaminación, envenenamiento y/o infección. Se hace notar que la práctica de lavar estos alimentos sólo antes de su consumo puede ser un factor de contaminación cruzada en la preparación y guardado de alimentos en el ambiente del hogar.

Merecen ser destacadas las similitudes predominantes entre ambientes urbano y rural en relación a los aspectos evaluados en este estudio, indicando que las principales vulnerabilidades observadas son comunes a la población del municipio, independientemente de la zona en la que residen.

Cabe señalar que dos factores indicaron para un mayor potencial de vulnerabilidad a la IAN en el medio rural en relación con el nivel socioeconómicos (la menor renta media del jefe de la familia) y de las condiciones de vida y salud (mayor frecuencia de uso de la fosa para eliminación de aguas residuales).

Se refuerza la importancia de los estudios que superar los enfoques tradicionales de la SAN, y que traten de este fenómeno en diferentes aspectos, incluyendo sus dimensiones variadas, ya que, según Habicht et al. (20), estos estudios pueden ser decisivos para el planeamiento de programas y políticas de carácter preventivo y de promoción de la SAN, consecuentemente, de la salud.

TABLA 3

Higienización de frutas y verduras por las familias de la zona urbana y rural de Chapecó (SC).

\begin{tabular}{lcc}
\hline & Población urbana (\%) & Población rural (\%) \\
Lavado de frutas & & 73,62 \\
Antes de comer & 26 & 25,58 \\
Antes de guardar & 0,30 & - \\
Las dos opciones & 0,7 & 2,79 \\
No lava & 76,47 & 76,27 \\
Lavado de verduras & 22,58 & 23,25 \\
Antes de comer & 0,45 & 0,48 \\
Antes de guardar & 0,50 & - \\
Las dos opciones & & \\
No lava & \\
\hline
\end{tabular}

Elaboración de los autores 


\section{CONCLUSIÓN}

Los resultados de este estudio demuestran que mientras algunas medidas sean adoptadas en la perspectiva de garantizar la Seguridad Alimentaria y Nutricional en la preparación, almacenamiento y consumo de alimentos, algunos aspectos aún necesitan mejorarse, como el destino de la basura y de las aguas residuales. El agua consumida por la población urbana y rural puede comprometer la salud ya que hay un gran número de ellas que utilizan agua de fuentes o pozos, especialmente si esta agua no es filtrada o vigilada su calidad. La eliminación inadecuada de basura y aguas residuales observados en este estudio puede comprometer las fuentes de abastecimiento de agua consumidos por la población urbana y rural.

Aunque la renta de las familias pueda posibilitar el acceso a la alimentación en cuantidad y calidad suficiente, se nota una desventaja, entre la población de la zona rural. Además, la educación de la mayoría de los jefes de familia puede representar un factor de vulnerabilidad a la IAN identificada en este estudio.

Estrategias básicas para garantizar la seguridad y la calidad en la producción de alimentos en los hogares son particularmente importantes, especialmente, las enfermedades transmitidas por los alimentos y las pérdidas económicas derivadas de un mal manejo.

El derecho humano a una alimentación, declarado y amparado por la ley, será asegurado cuando la sociedad tenga la conciencia sobre los riesgos a la salud y al medio ambiente, causados por prácticas inadecuadas desde la producción hasta el consumo de alimentos.

\section{RESUMEN}

El objetivo de este trabajo fue conocer las condiciones socioeconómicas, higiénico-sanitarias y el saneamiento básico de los hogares del municipio de Chapecó (SC) como dimensiones de la Seguridad Alimentaria y Nutricional. Fueron investigados 774 hogares en las zonas urbana y rural. La mayoría de los jefes de los hogares eran mujeres (68,8\%). La renta media familiar mensual era 1.803,8 dólares. El sistema de alcantarillado público sólo existe en la zona urbana $(23,4 \%)$. Se considera que la renta familiar observada mejora el acceso a la alimentación; Sin embargo, el nivel de educación de más de $70 \%$ de los jefes es igual o inferior a la educación primaria. Esto puede implicar la falta de cuidado con la higienización y manipulación de los alimentos. El gran número de familias que utiliza agua de fuentes/pozos para el consumo humano puede perjudicar su condición de Seguridad Alimentaria y Nutricional.

Palabras clave: Indicadores socioeconómicos, seguridad alimentaria y nutricional, urbano, rural.

Financiamiento: FAPESC - Fundação de Amparo à Pesquisa e Inovação do Estado de Santa Catarina (processo $\mathrm{n}^{\circ}$ 2.443/2011-0).

FAPE - Fundo de Apoio à Pesquisa da Universidade Comunitária da Região de Chapecó (UNOCHAPECÓ). (Editais 025/REITORIA/2010 e 036/REITORIA/2011).

\section{BIBLIOGRAFÍA}

1. Brasil. Conselho Nacional de Segurança Alimentar. Princípios e diretrizes de uma política nacional de segurança alimentar e nutricional. Texto de referência da II Conferência Nacional de Segurança Alimentar e Nutricional. Brasília: CONSEA; 2004.

2. Herrán OF. Quintero DC, Prada GE. Validez factorial, con- sistencia interna y reproducibilidad de la escala de seguridad alimentaria en hogares de Bucaramanga, Colombia. Rev Chil Nutr. 2009;36:169-79.

3. Cardoso RCV, Souza EVA, Santos PQ. Unidades de alimentação e nutrição nos campi da Universidade Federal da Bahia: um estudo sob a perspectiva do alimento seguro. Rev Nutrição 2005;18:669-80.

4. Akutsu RC, Camargo EB, Sávio KEO, Araújo WC. Adequação das boas práticas de fabricação em serviços de alimentação. Rev Nutrição 2005;18:419-27.

5. Marchi DM, Baggio N, Busato MA, Teo CRPA. Ocorrência de surtos de doenças transmitidas por alimentos no Município de Chapecó, Estado de Santa Catarina, Brasil, no período de 1995 a 2007. Epidemiol Serviços Saúde 2011;20:401-07.

6. Segall-Corrêa AM, Escamilla RP, Maranha LK, Sampaio MFA. Projeto Acompanhamento e avaliação da segurança alimentar de famílias brasileiras: validação de metodologia e de instrumento de coleta de informação. Campinas: Departamento de Medicina Preventiva e Social, Universidade Estadual de Campinas/Organização Pan-Americana da Saúde/ Ministério de Saúde; 2004.

7. Instituto Brasileiro de Geografia e Estatística (IBGE). Pesquisa Nacional por amostra de domicílios - PNAD. Segurança Alimentar: 2004. Rio de Janeiro: IBAMA; 2006.

8. Piragine $K O$. Aspectos higiênicos e sanitários do preparo da merenda escolar na rede estadual de ensino de Curitiba. [Dissertação] Curitiba. Universidade Federal do Paraná; 2005.

9. Panigassi G, Segall-Corrêa AM, Marín-León L, PérezEscamilla R, Sampaio MFA, Maranha LK. Insegurança alimentar como indicador de iniqüidade: análise de inquérito populacional. Cadernos Saúde Pública 2008; 24: 2376-84.

10. Siliprandi E. Políticas de segurança alimentar e relações de gênero. Cadernos Debates 2004;11:38-57.

11. Marín-León L, Francisco PMSB, Segall-Corrêa AM, Panigassi $G$. Bens de consumo e insegurança alimentar: diferenças de gênero, cor de pele autorreferida e condição socioeconômica. Rev Brasileira Epidemiol. 2011;14:398410.

12. Salles-Costa $R$, Pereira $R A$, Vasconcellos $M T L$, Veiga $G V$, Marins VMR, Jardim BC, Gomes FS, Sichieri $R$. Associação entre fatores socioeconômicos e insegurança alimentar: estudo de base populacional na Região Metropolitana do Rio de Janeiro, Brasil. Rev Nutrição 2008; 21: 99-109.

13. Lozano CG, Estrada RA. Seguridad alimentaria en hogares de Acandí, Darién Caribe Colombiano: el aporte del caracol Cittarium pica "la cigua". Rev Chil Nutr. 2009; 35: 460-70.

14. Panigassi G. Inquérito populacional sobre a percepção da segurança alimentar intrafamiliar no município de Campinas. [Tese] Capinas. Universidade Estadual de Campinas/ UNICAMP; 2005

15. Azeredo CM, Cotta RMM, Schott M, Maia TM, Marques ES. Avaliação das condições de habitação e saneamento: a importância da visita domiciliar no contexto do Programa de Saúde da Família. Ciência Saúde Coletiva 2007; 12:743-53.

16. Amaral LA, Nader Filho A, Rossi Junior $O D$, Ferreira $L A F$, Barros LSS. Água de consumo humano como fator de risco à saúde em propriedades rurais. Rev Saúde Pública 2003:37:510-4. 
17. Amorim L, Kuhn M, Blank VL, Gouveia N. Saúde Ambiental nas cidades. Tempus - Actas Saúde Coletiva 2009;4:111-20.

18. Baptista $P$, Antunes C. Higiene e segurança alimentar na restauração. v. Il Avançado. Guimarães: Forvisão, Portugal. 2005.

19. Oliveira MN, Brasil ALD, Taddei JAAC. Avaliação das condições higiênico-sanitárias das cozinhas de creches públicas e filantrópicas. Ciência Saúde Coletiva 2008;13:1051-60.

20. Habicht JP, Pelto G, Frongillo E, Rose D. Conceptualization and instrumentation of food insecurity. National Academy of Sciences Workshop [cited 2007 Jun 13]. Disponível em: http://www7.nationalacademies.org/cnstat/ Conceptualization_and_Instrumentation_of_Food_Security_Paper.pdf 